\title{
Innovación educativa y gestión del conocimiento
}

Oscar Yecid Aparicio Gómez y Olga Lucía Ostos Ortiz Editores académicos

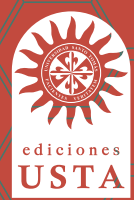




\title{
Nuevas competencias del profesorado STEM: un modelo de formación
} en línea basado en procesos de indagación

\author{
MARIO BARAJAS FRUTOS \\ RICARDO TORREBLANCA ROJAS \\ Silvia AlCARAZ DOMÍNGUEZ
}

$\mathrm{E}$ n este capítulo se propone un modelo de desarrollo y consolidación de las competencias del profesorado de ciencias experimentales, tecnología y matemáticas (STEM, por sus siglas en inglés) en los niveles de enseñanza secundaria y bachillerato. La propuesta está basada en el análisis de programas de formación inicial de profesorado STEM y de las políticas educativas vigentes promovidas en la Unión Europea, que responden a los retos que enfrenta la enseñanza de las ciencias en el contexto educativo actual. Más allá de analizar cómo se aborda la enseñanza de los contenidos de las diferentes materias STEM, se presenta una estructura de desarrollo de competencias del profesorado, que introduce la metodología del aprendizaje basado en la indagación, con contenidos socio-científicos que puedan ser más atractivos para el alumnado actual y que complementen su currículum. 
La investigación que sustenta este capítulo fue realizada dentro del proyecto internacional ELITe - financiado por la Unión Europea- ${ }^{1}$, que se propone mejorar las competencias del profesorado STEM mediante una metodología de capacitación que impulse el desarrollo de habilidades reflexivas y de investigación a través del aprendizaje basado en la indagación, utilizando una plataforma digital abierta para la formación denominada DojoIBL.

\section{Introducción}

La educación en STEM cumple en nuestra sociedad una función clave para el desarrollo económico. Informes recientes resaltan el hecho de que el crecimiento basado en la innovación produce puestos de trabajo con un valor añadido (Corlu, 2014).

Dado que la innovación implica integrar diversas competencias STEM, trascender las disciplinas y establecer un proceso altamente interactivo y multidisciplinario, la importancia de la educación STEM aumenta dramáticamente para la economía y la sociedad actualmente. Este campo del saber se conecta con el desarrollo de conocimientos, conceptos y prácticas científicas, tecnológicas y matemáticas, así como con la forma de utilizarlos y aplicarlos para resolver cuestiones complejas o desafíos de la vida real. Además, la educación STEM potencia el pensamiento analítico y crítico, y las competencias de investigación, cruciales para promover una mentalidad innovadora en las generaciones actuales. Según el informe de la Comisión Europea "Science Education for Responsible Citizenship” (European Commission, 2015), la educación STEM es vital en el siglo XXI para lograr, entre otros objetivos:

1 ELITe (Enhancing Learning in Teaching via e-inquiries) es un proyecto europeo, financiado por el programa ERASMUS+ de la Unión Europea, Key Action 2 (2016-2019). Agradecimiento al n. $2016-1-E L 01-K A 201-023647$. Para más información ver http://www.learning-in-teaching.eu/index.php/es/ 
- Promover una cultura de pensamiento científico e inspirar a los ciudadanos a utilizar el razonamiento basado en la evidencia en la toma de decisiones.

- Garantizar que los ciudadanos tengan la confianza, los conocimientos y las competencias necesarias para participar activamente en un mundo científico y tecnológico cada vez más complejo.

- Desarrollar las competencias en resolución de problemas y en innovación, así como el pensamiento analítico y crítico que son tan necesarios para capacitar a los ciudadanos en una vida satisfactoria personalmente, socialmente responsable y profesionalmente comprometida.

- Inspirar a los estudiantes de todas las edades y talentos a cursar carreras científicas y a otras ocupaciones y profesiones que promueven economías intensivas en conocimiento e innovación, en las que pueden ser creativos y lograr sus objetivos.

En resumen, la educación STEM tiene el potencial de ayudar a los jóvenes a interpretar y comprender nuestro mundo, a gestionar riesgos con un componente de incertidumbre, a guiar el desarrollo tecnológico y la innovación, y a pronosticar y planear el futuro. La educación STEM puede mejorar las perspectivas de empleo, la conciencia cultural y la capacidad de actuar bien informados en solidaridad con los ciudadanos de todo el mundo.

Teniendo en cuenta esta amplia visión de STEM en la sociedad actual, nadie puede dudar de la importancia de la formación del profesorado en este campo. En este capítulo se propone un modelo de desarrollo y consolidación de las competencias del profesorado STEM basado en el análisis de programas de formación inicial de profesorado y en las políticas educativas vigentes promovidas en la Unión Europea, que contempla los retos a los que se enfrenta la enseñanza de las ciencias en el contexto educativo actual. Más que centrarse en 
cómo se aborda la enseñanza de los contenidos de las diferentes materias STEM, se presenta una estructura de desarrollo de competencias del profesorado, utilizando la metodología del aprendizaje basado en la indagación (IBL, por sus siglas en inglés), y en contenidos socio-científicos que puedan ser más atractivas para el alumnado actual y complementar el currículum. El fin último de las reflexiones presentadas en este capítulo es conseguir que el alumnado de la enseñanza obligatoria se sienta atraído por los estudios STEM, dado que el desinterés en este campo es un problema que se ha agudizado en muchos países; despertar esta inquietud promovería, además de las ventajas mencionadas, un mejor rendimiento en sus aprendizajes. Desde hace años se observa una carencia en la demanda de profesionales STEM, teniendo en cuenta las necesidades de una sociedad tecnológica, así como una disminución de estudiantes universitarios, especialmente mujeres, quienes, excepto en las áreas relacionadas con ciencias de la salud, no tienen una representación adecuada a su población (Dick y Rallis, 1991; Rossi y Barajas, 2015).

La metodología de aprendizaje basado en la indagación comenzó a consolidarse hace años como una de las opciones que proponen cambiar la perspectiva actual de la enseñanza y el aprendizaje, como indica el informe "Science Education Now: A Renewed Pedagogy for the Future of Europe" (European Commission, 2007). Las propuestas y resultados del proyecto internacional ELITe se dieron gracias al diseño e implementación de ocho módulos para la formación docente STEM en cuatro países de la Unión Europea (Grecia, Holanda, Bulgaria y España), cuya estructura está específicamente adaptada para aplicar la metodología de enseñanza por indagación, en la que se exploran individual y colaborativamente los diversos temas presentados. Por esta razón, consideramos que la combinación de nuevos contenidos sociocientíficos de tipo transversal con la metodología de indagación puede mejorar las competencias docentes del profesorado STEM, e inspirar la actualización de los programas de desarrollo profesional en este campo del conocimiento. 


\section{Aprendizaje basado en la indagación aplicado a STEM}

El aprendizaje por indagación es considerado como una metodología adecuada en la formación del profesorado y para su utilización en el aula. Es una metodología de enseñanza y aprendizaje activo que implica, en general, realizar observaciones, formular preguntas, consultar bibliografía y otras fuentes de información para entender qué conocimiento existe y revisarlo mediante la evidencia científica, planificar investigaciones, realizar experimentos, utilizar herramientas para recoger, analizar e interpretar datos, proponer respuestas, explicaciones y predicciones, y comunicar los resultados (Kapelari et al., 2012). Aunque las definiciones pueden variar, hay un acuerdo en que su característica central es el énfasis en la pregunta de investigación como orientadora de las experiencias de aprendizaje de los estudiantes (Crawford, 2000; Cuevas et al., 2005; Deters, 2004). También se hace hincapié en que los profesores participen en investigaciones colaborativas (Bell et al., 2010). Además, el “aprendizaje guiado por la indagación fomenta la adquisición de nuevos conocimientos, habilidades y actitudes a través de preguntas, problemas y controversias, utilizando los estándares de investigación de las diferentes disciplinas" (Lee, 2011).

En este sentido, se ha demostrado que el modelo guiado de IBL es más efectivo, dado que los estudiantes mejoran sus resultados respecto a modelos más abiertos (Lazonder y Harmsen, 2016). Si utilizamos tecnologías, los resultados de este proceso colaborativo pueden ser muy fructíferos (Van Joolingen et al., 2005).

Las actividades se pueden desarrollar de manera individual o colaborativa, y el proceso puede estar orientado por el profesor al tiempo que el alumnado desarrolla su propia iniciativa en la planificación y desarrollo de su investigación. En el modelo guiado, la pregunta que inicia el proceso de indagación está relacionada con temáticas sobre las cuales el estudiante habrá de plantear nuevas preguntas para continuar la indagación. 
El aprendizaje basado en la indagación en STEM implica:

- Observar: mirar con atención, tomar notas, comparar y contrastar.

- Cuestionar: formular preguntas sobre las observaciones que pueden dar lugar a investigaciones.

- Aceptar o rechazar hipótesis: elaborar explicaciones coherentes con las observaciones realizadas.

- Investigar: planificar, llevar a cabo acciones, tomar medidas, recopilar datos, controlar variables.

- Interpretar: sintetizar, extraer conclusiones, identificar patrones.

- Comunicar: informar a los demás mediante varios medios: oral, escrito y de representación.

- Evaluar: elaborar opiniones críticas basadas en observaciones y conocimientos ya adquiridos.

Una pedagogía IBL para la enseñanza STEM implica, en primer lugar, que el profesor pueda alimentar un diálogo persuasivo con el alumnado mediante la formulación de preguntas auténticas, es decir, aquellas que tienen que ver con su realidad circundante, basadas en las temáticas actuales que puedan atraer su interés por la ciencia. Este proceso permitirá plantear retos a los estudiantes, en los cuales el profesor puede cuestionar las concepciones erróneas formulando hipótesis refutables mediante una investigación. También es clave la reflexión (por ejemplo, a partir de establecer comparaciones, conflictos, etc.) para despertar el interés por saber cómo funciona un fenómeno estudiado; así como realizar la observación completa de ese fenómeno y formular una hipótesis, que permita el razonamiento y la verificación mediante la investigación empírica. 


\section{Algunas limitaciones prácticas para la implementación de IBL en STEM}

Existe un consenso en la necesidad de mejorar la calidad de la educación científica tanto de los estudiantes, los futuros graduados, el profesorado y la sociedad en general. Adoptar la metodología de aprendizaje por indagación podría ser una manera de hacerlo, aunque su aceptación en la educación es un reto que debe superar muchos problemas. En un estudio europeo realizado con profesores y expertos de catorces países (Barajas et al., 2011) se identificó un conjunto de obstáculos comunes a superar en la utilización de IBL en STEM:

- La falta de experiencia y formación en metodologías IBL del profesorado con pocos recursos adecuados para esta metodología.

- Los profesores que implementan IBL muchas veces trabajan individualmente y rara vez colaboran con otros colegas, o no son apoyados por las direcciones de los centros.

- Los planes de estudios están orientados a los contenidos y tienen una fuerte dependencia de los libros de texto.

- El número de estudiantes por clase y la falta de tiempo para la preparación e implementación de actividades desalienta al profesorado a realizar estas actividades que requieren generalmente mayor esfuerzo.

- Una evaluación basada en contenidos, sumada a la presión de los exámenes de ingreso a la universidad que aún se basan en el dominio de los contenidos.

- La incompatibilidad entre los horarios escolares actuales y el tiempo requerido para las actividades de aprendizaje basadas en indagación. 
En cualquier programa de formación del profesorado, sea inicial o permanente, estas dificultades se han de considerar desde un punto de vista práctico para su superación, de forma que los profesores no experimenten las ya conocidas frustraciones de implementar metodologías innovadoras en contextos reales que no corresponden con los de la investigación académica o el laboratorio.

\section{El proyecto ELITe para la formación del profesorado STEM}

La capacitación del profesorado STEM y el apoyo al desarrollo de prácticas IBL en el aula se consideran esenciales en el proceso de implementación de la metodología. Muchas veces se ha dicho que el profesor enseña de la manera en que se le enseñó. Por lo tanto, la buena formación del profesorado es crucial, especialmente aquella que permita al profesor ponerse en el lugar del estudiante. El aprendizaje basado en la indagación se ha identificado como un enfoque de enseñanza innovador, que brinda oportunidades para desarrollar la alfabetización científica del alumnado. Al mismo tiempo, como ya se ha comentado, los profesores encuentran dificultades para implementarlo en el aula, debido a la falta de experiencia, ya que, por lo general, los cursos de formación docente se llevan a cabo de manera tradicional. El supuesto principal del proyecto ELITe es que la implementación de la metodología IBL en el desarrollo de competencias de los docentes les proporcionará experiencia y conocimientos de la situación real, pero también como una reflexión desde el punto de vista de los estudiantes. Por otro lado, la metodología IBL se ha explorado poco en el campo de la capacitación docente que puede contribuir al desarrollo efectivo de la competencia de los docentes STEM.

A menudo los profesores creen estar familiarizados con IBL, pero cuando se les pide que los discutan, se revela que lo entienden más bien como un enfoque de resolución de problemas o simplemente como una formación práctica, en la que los estudiantes realizan experimentos, pero sin exigir que formulen y revisen explicaciones científicas. Algunos profesores conocen aspectos de la metodología, tales como 
los conceptos de pregunta de investigación o reto inicial, pero probablemente están menos familiarizados con procesos más complejos, tales como las preguntas de investigación propias de los estudiantes. Además, aunque los profesores están familiarizados con una función de facilitador, persiste una tendencia hacia un tipo de enseñanza más dirigida, cuando no centrada en contenidos. La formación del profesorado STEM en IBL debería responder a esos problemas. Desarrollar una comprensión completa e informada del enfoque IBL, de sus beneficios y de las formas de implementarlos, es esencial, es decir, un profesorado bien informado, capacitado y apoyado (con materiales y otros recursos) puede impulsar un cambio significativo en la práctica. También se requiere apoyo técnico, ya que esto puede facilitar que los profesores prueben nuevas metodologías y recursos.

En este sentido, a diferencia de la formación tradicional del profesorado, el proyecto ELITe promueve un modelo flexible de desarrollo del docente STEM, que considera al profesorado como un profesional reflexivo y responsable de su propio aprendizaje, fundamentado en las siguientes premisas:

- Adoptar un enfoque adaptado al entorno nacional/local para el desarrollo profesional STEM, teniendo en cuenta los requisitos de las políticas y las necesidades prácticas del sistema educativo de cada país.

- Promover la implementación de una metodología IBL en el desarrollo profesional, bajo la premisa de que la capacitación de los docentes STEM con esta metodología mejora el desarrollo de sus competencias profesionales, especialmente si se hace a través de procesos formativos colaborativos.

- Proponer áreas de contenido temático STEM que reflejan las políticas y prioridades educativas actuales, con objetivos más amplios, de tipo sociocientífico, para la educación STEM, con el propósito de facilitar el desarrollo de las competencias del profesorado y, por ende, ayudar a los estudiantes a adquirirlas. 
ELITe combina el enfoque IBL en la formación del profesorado con un modelo de práctica reflexiva. En la línea de Barnet y O’Mahey (2006), la práctica reflexiva se caracteriza por el análisis retrospectivo de la propia práctica, que considera las posibilidades de mejora o de acercamiento a una situación deseada. Para ello se exploran diversas posibilidades y se toman decisiones basadas en evidencias. Por último, se evalúan los resultados de la implementación, de manera que se cierra la primera etapa para comenzar un nuevo ciclo reflexivo. Así pues, el enfoque propuesto considera cuatro niveles de indagación y de práctica reflexiva (figura 1).

Figura 1. Niveles de indagación y práctica reflexiva STEM

Indagación y prácticas de enseñanza reflexiva de los formadores del profesorado STEM

Indagación y prácticas de aprendizaje reflexivo del profesor STEM

Indagación y prácticas de enseñanza reflexiva del profesor STEM
Práctica profesional del profesor

Fuente: elaboración propia.

La indagación y práctica reflexiva de los formadores tiene lugar: 1) en una primera fase, al estudiar los requisitos implícitos y explícitos de las competencias del profesorado STEM; 2) durante el diseño de la formación, al reflexionar sobre esos requisitos, y también al ofrecer un modelo de metacurso que pueda ser usado por los profesores en su práctica docente; 3) durante la formación, como método facilitador; y 4) como una reflexión posterior a la formación para evaluar qué se aprendió y cómo se podría mejorar el siguiente proceso formativo. 
La práctica del profesorado STEM, por otra parte, considera los roles de los profesores, según el modelo de aprendizaje a lo largo de la vida, como facilitadores del aprendizaje de los estudiantes (práctica de enseñanza y aprendizaje) y como miembros de comunidades educativas profesionales (práctica profesional). La indagación y las prácticas de aprendizaje reflexivo ayudan al profesor a ser consciente de lo aprendido por él mismo, y por el trabajo colaborativo y experiencias compartidas con otros docentes. Todos estos aspectos están integrados en el modelo de ELITe de IBL para el desarrollo de competencias del profesorado STEM en forma de discusiones, reflexiones y retroalimentación continua por y entre los participantes.

El enfoque de ELITe de aprendizaje en la enseñanza vía indagación en la plataforma DojoIBL, que veremos a continuación, anima a los profesores a la autorreflexión durante su práctica, de modo que puedan identificar aspectos problemáticos y actuar como estudiantes cuando eligen cursos de formación relevantes. En este modelo, la comunicación es también una parte integral del desarrollo de competencias de los profesores.

\section{La plataforma digital DojoIBL}

El sistema en la nube DojoIBL es la plataforma digital básica para la formación adoptada en el proyecto. Fue desarrollada por la Universidad Abierta de Holanda (oun, socia de ELITe) para su aplicación como entorno de formación híbrida o a distancia. La plataforma ayuda a los formadores de docentes a aplicar procesos de aprendizaje colaborativo, tales como el IBL o el aprendizaje basado en los problemas $(\mathrm{PBL})$. En la plataforma los formadores pueden crear (o reutilizar) estructuras de aprendizaje IBL y organizar múltiples grupos de profesores para trabajar en ellas.

Los profesores en formación (y en su caso sus estudiantes, si el profesor diseña actividades de aprendizaje y las implementa en el aula) pueden colaborar y hacer uso de las diferentes funcionalidades para seguir su progreso individual y en grupo. La interfaz de usuario es simple pero potente, y está traducida a varios idiomas, incluido 
el español. Además, el contenido de DojoIBL se puede integrar en otros entornos de e-learning específicos de la institución que implementa la formación del profesorado, e incluir una variedad de herramientas TIC apropiadas para una actividad específica de desarrollo de habilidades.

Para utilizar DojoIBL cada usuario debe iniciar su sesión a través de una cuenta de Google o mediante el registro de una cuenta nueva utilizando su correo electrónico.

Para participar en un módulo determinado como alumno es preciso introducir el código correspondiente, recibido del formador (ver anexo 1).

En cuanto a la creación de módulos con DojoIBL, la plataforma permite reutilizar módulos ya creados e incluso modificarlos. Pero lo más importante es que el formador puede crear sus propios escenarios de aprendizaje. Para ello puede utilizar una estructura de IBL ya creada y completarla con contenido y actividades, o puede crear la suya propia desde el principio.

En caso de que se prefiera crear un módulo desde cero, DojoIBL es un sistema abierto, en el sentido de que a partir de una estructura de IBL vacía, se pueden diseñar módulos o actividades de aprendizaje siguiendo las diferentes modalidades de IBL, desde la más abierta (el alumno plantea el problema y la pregunta de indagación), hasta la más dirigida (el profesor diseña todas las etapas de la indagación), pasando por otras modalidades intermedias. Para ello, basta que el profesor escoja o descarte las etapas deseadas de la indagación, o utilice plantillas completas ya construidas, en el último caso.

\section{La propuesta ELITe de desarrollo de competencias del profesorado STEM}

Parece haber una variedad de enfoques en los requisitos de las competencias del profesorado en las políticas educativas nacionales de la Unión Europea, que van desde una definición "ligera" de conjuntos amplios en los marcos de competencias hasta listas prescriptivas, relacionadas con las normas profesionales y la promoción de la carrera 
profesional (Caena, 2011). Sin embargo, los principales aspectos de las competencias del profesorado, tal como se definen en el marco de la Comisión Europea (European Commission, 2013), son más o menos evidentes en los contextos nacionales de desarrollo profesional de los docentes de STEM.

La indagación y la práctica reflexiva en la formación del profesorado STEM resultan, en primer lugar, en una evaluación crítica del contexto local/nacional de enseñanza, que aporta flexibilidad a los programas de desarrollo profesional del profesorado. Para ello, se han de estudiar las políticas nacionales sobre las competencias de los docentes en cada país, de manera que se pueda definir un plan estratégico para el desarrollo profesional de los educadores. Se trata de un marco holístico que tiene como objetivos principales:

- Estructurar la capacitación del profesorado (inicial y en servicio) en torno a un nuevo modelo de competencias profesionales para la educación del siglo XxI.

- Explorar nuevas modalidades de formación que faciliten la colaboración entre profesionales de la enseñanza.

- Establecer un marco normativo que permita la certificación de las competencias profesionales y de las actividades que demuestren un desarrollo profesional efectivo para docentes y formadores.

Para ELITe este marco está compuesto por tres pilares principales: 1) las competencias profesionales de los docentes (que se proponen redefinir el perfil de los profesionales de la enseñanza, mediante un marco de competencias que permita a los docentes desarrollar y evaluar las competencias de los estudiantes);2) las nuevas modalidades de capacitación (las cuales procuran incorporar aprendizajes, por ejemplo, en las comunidades de práctica en línea y en otras situaciones de aprendizaje); y 3) la certificación de la capacitación (que actualiza, desde el punto de vista de la competencia, las regulaciones para la certificación de la capacitación docente). 
Los escenarios identificados para la formación y actualización de los docentes STEM corresponden a áreas temáticas relacionadas con los desafíos actuales. Mediante una revisión de estudios de la oECD (2013, 2018), como TALIS, que ofrecen interesantes visiones sobre la percepción del profesorado de las necesidades y participación en la formación continua docente (CPD), así como de la negociación con expertos educativos en los países participantes en ELITe sobre las áreas de contenido más relevantes para cada país. Finalmente, se consensuaron en el proyecto las áreas potenciales que deberían tratarse en la formación del profesorado STEM:

- Inclusión y diversidad en STEM.

- Enseñanza STEM para el desarrollo de habilidades.

- Incorporación de RRI (investigación e innovación responsable) en la educación STEM.

- Aplicación de metodologías didácticas innovadoras (IBL y trabajo de proyectos, aprendizaje autodirigido, pensamiento computacional, etc.) para STEM.

- Vincular los centros educativos con la ciencia y la investigación.

- Asumir los retos de la evaluación en STEM.

- Usar tecnologías y recursos digitales para mejorar la enseñanza y el aprendizaje STEM.

- Resolver los retos del nuevo currículum STEM.

- Mejorar la colaboración profesorado-familias en el aprendizaje STEM. 


\section{El espacio de intervención ELITe en España en la formación del profesorado STEM}

El modelo ELITe ha sido aplicado en cuatro países europeos durante el periodo 2018-2019. Como ejemplo de diseño y aplicación del modelo, a continuación, analizamos el espacio de intervención para formación y desarrollo profesional de los docentes de STEM en España. Este ejemplo puede servir como modelo metodológico para realizar estudios semejantes en otros países.

\section{Las políticas educativas en España}

Las leyes educativas españolas han sido cambiadas varias veces durante los últimos veinte años. Desde 2013, existe una nueva ley educativa denominada LOMCE, Ley Orgánica para la Mejora de la Calidad de la Educación (вов, 2013), del Ministerio de Educación. Fruto de la falta de consenso, esta Ley ha sido impugnada en muchos niveles, lo que le ha dado un sentido de provisionalidad que se refleja en el hecho de que las regiones, las autoridades locales y las instituciones educativas estén en negociaciones con el Ministerio para modificarla. En esta situación hay cierto cansancio frente a los continuos cambios jurídicos y, también, recortes presupuestarios en educación.

Sin embargo, en la Ley aparecen cambios importantes en concordancia con las políticas de la Unión Europea (Parlamento Europeo, 2006). Se enfatizan la calidad y la empleabilidad para fomentar la futura selección tanto de la trayectoria profesional como de la académica en la etapa de secundaria. La Ley prioriza en STEM, en disciplinas más instrumentales, y menos en asignaturas opcionales. Es importante el interés de ofrecer a las escuelas más libertad en cuanto a la adaptación del currículo nacional (propuesta curricular) o en introducir nuevas metodologías y asignaturas optativas (programa didáctico), dentro de sus competencias legales. El currículo se organiza de acuerdo con un enfoque competencial, en el que se han identificado los conocimientos, habilidades y actitudes en todas las asignaturas y en todos los niveles. Las competencias clave de la Unión Europea, denominadas “competencias básicas”, conforman el modelo planteado. Este panorama 
implica también la reforma de los programas de desarrollo profesional del profesorado, así como el acceso a la profesión docente, basado en la realización de un programa oficial de máster.

\section{La práctica docente y los currículums STEM de secundaria}

En la práctica docente, la LOMCE prioriza el desarrollo de las competencias de los estudiantes. Estas se encuentran presentes en el desarrollo curricular y en la evaluación de todos los niveles y modalidades educativas. Además, la Ley establece que las habilidades cognitivas deben ir acompañadas de la adquisición de competencias transversales por parte de los estudiantes, como el pensamiento crítico, la gestión de la diversidad, la creatividad y las habilidades de comunicación. Las competencias básicas se introdujeron en 2007 por primera vez en el Currículo Nacional de Educación Secundaria de España, con grandes similitudes con las establecidas en el Marco de Referencia Europeo (Parlamento Europeo, 2006): comunicación lingüística, competencia matemática, competencia en el conocimiento e interacción con el mundo físico, competencia digital y de procesamiento de la información, competencia social y ciudadana, competencia cultural y artística, aprendizaje para aprender, autonomía personal e iniciativa. Posteriormente, se desarrolló la relación entre las competencias, los contenidos y los criterios de evaluación en los niveles de educación primaria, secundaria y bachillerato (BOE, 2015).

En los planes de estudio se establece que los estudiantes deben adquirir el nivel adecuado de las competencias al final de la educación obligatoria. Además, explica cómo cada materia contribuye a la adquisición de las competencias antes mencionadas. Una revisión de los planes de estudio de STEM de los estudiantes en el país evidencia que se requieren implícitamente los siguientes aspectos de las competencias del profesorado STEM en el país:

- Conocimiento y comprensión: conocimiento del contenido pedagógico; inclusión y diversidad; uso efectivo de las tecnologías en el aprendizaje. 
- Habilidades: recopilación de análisis, interpretación de evidencias y datos para mejorar la enseñanza/aprendizaje; uso, desarrollo y creación de conocimientos de investigación para informar prácticas.

- Disposiciones y actitudes: disposiciones para promover las actitudes y prácticas democráticas de los estudiantes como ciudadanos europeos (incluida la apreciación de la diversidad y la multiculturalidad); disposición al trabajo en equipo, colaboración y creación de contactos.

\section{Mecanismos de mediación: la formación de los docentes STEM}

En la actualidad, las universidades se han enfrentado a un importante desafío para crear programas de máster para la formación de profesorado de educación secundaria según un enfoque competencial (centrado en aspectos tales como los conocimientos, las habilidades y las actitudes del alumnado). En general, las competencias que se destacan para la acreditación de docentes en materias relacionadas con STEM Son:

- Conocimiento y comprensión: conocimiento de la materia; conocimiento pedagógico; conocimiento curricular; fundamentos de la ciencia educativa; aspectos contextuales, institucionales y organizativos de las políticas educativas; temas de inclusión y diversidad; uso efectivo de las tecnologías en el aprendizaje; psicología del desarrollo; aprendizaje de teorías y cuestiones motivacionales; evaluación y valoración.

- Habilidades, tales como: planificación, gestión y coordinación de la enseñanza; uso de materiales y tecnologías de enseñanza; gestión de alumnos y grupos; uso, desarrollo y creación de conocimiento para informar la práctica; promoción de habilidades reflexivas metacognitivas; y adaptación a los contextos educativos. 
- Disposiciones y actitudes: conciencia epistemológica; habilidades de enseñanza a través del contenido; disposición al cambio, flexibilidad; compromiso para promover el aprendizaje de todos los estudiantes; disposición para promover actitudes democráticas estudiantiles; actitudes de trabajo en equipo.

Cualquier innovación en la formación docente ha de tener en cuenta los programas y competencias actuales de los másteres oficiales de las universidades españolas. Es evidente que la propuesta ELITe no puede entrar en contradicción con estos. Es evidente que será más fácil introducir nuevos contenidos y metodologías didácticas cuando se trata de programas para la actualización y formación del profesorado STEM en servicio.

\section{El desarrollo de competencias del profesorado STEM en España}

En este contexto, siguiendo el modelo ELITe de localización según regiones o países, se han identificado requisitos y contenidos temáticos para el desarrollo de las competencias del profesorado STEM, basados en el análisis de las competencias, presentado en la sección anterior, y del currículum STEM de secundaria.

La tabla 1 ofrece una visión general de las competencias de los docentes STEM que se requieren en el modelo ELITe y que están presentes en el contexto nacional español, sin menoscabo de algunas diferencias a nivel regional. Se tienen en cuenta tres dimensiones de las competencias (conocimiento y comprensión; habilidades; disposiciones y actitudes), mientras que los aspectos que caracterizan a cada dimensión han sido adoptados del marco de competencias docentes de la Unión Europea (European Commission, 2013). Estos aspectos de cada dimensión se consideran explícitos si hay evidencias en los documentos y planes de estudio de la formación de docentes; los aspectos implícitos se refieren a las evidencias mostradas en los currículos de los estudiantes. 
Tabla 1. Aspectos implícitos y explícitos de las competencias del profesorado en España

\begin{tabular}{|l|l|l|}
\hline \multicolumn{1}{|c|}{ Conocimiento y comprensión } & Explícito & Implícito \\
\hline Conocimiento de la materia & & \\
\hline Conocimiento del contenido pedagógico & & \\
\hline Conocimiento pedagógico & & \\
\hline Conocimiento del currículo & & \\
\hline Fundamentos de la ciencia educativa & & \\
\hline $\begin{array}{l}\text { Aspectos contextuales, institucionales, organizativos } \\
\text { de las políticas educativas }\end{array}$ & & \\
\hline Temas de inclusión y diversidad & & \\
\hline Uso efectivo de tecnologías en aprendizaje & & \\
\hline Psicología del desarrollo & & \\
\hline $\begin{array}{l}\text { Procesos y dinámicas grupales, teorías de } \\
\text { aprendizaje, cuestiones motivacionales }\end{array}$ & & \\
\hline Evaluación y valoración & & \\
\hline
\end{tabular}

\begin{tabular}{|l|l|l|}
\hline \multicolumn{1}{|c|}{ Habilidades } & Explícito & Implícito \\
\hline Planificación, gestión y coordinación de la docencia & & \\
\hline Uso de materiales docentes y tecnologías & & \\
\hline Gestión de estudiantes y grupos & & \\
\hline $\begin{array}{l}\text { Seguimiento de la adaptación y evaluación de los } \\
\text { objetivos y procesos de enseñanza y aprendizaje }\end{array}$ & & \\
\hline $\begin{array}{l}\text { Recoger, analizar, interpretar evidencias y datos para } \\
\text { la toma de decisiones profesionales }\end{array}$ & & \\
\hline $\begin{array}{l}\text { Uso, desarrollo y creación de conocimiento de } \\
\text { investigación para prácticas actualizadas }\end{array}$ & & \\
\hline
\end{tabular}




\begin{tabular}{|l|l|l|}
\hline \multicolumn{1}{|c|}{ Habilidades } & Explícito & Implícito \\
\hline $\begin{array}{l}\text { Colaboración con colegas, padres y madres y } \\
\text { servicios sociales }\end{array}$ & & \\
\hline $\begin{array}{l}\text { Habilidades reflexivas, metacognitivas e } \\
\text { interpersonales para el aprendizaje individual y en } \\
\text { comunidades profesionales de práctica }\end{array}$ & & \\
\hline Adaptación a contextos educacionales & & \\
\hline
\end{tabular}

\begin{tabular}{|l|l|l|}
\hline \multicolumn{1}{|c|}{ Disposición y actitudes } & Explícito & Implícito \\
\hline Conciencia epistemológica & & \\
\hline Habilidades para enseñar el contenido concreto & & \\
\hline Habilidades de transferencia & & \\
\hline $\begin{array}{l}\text { Disposición de cambio, flexibilidad, mejora continua } \\
\text { del aprendizaje profesional, incluyendo estudio e } \\
\text { investigación }\end{array}$ & & \\
\hline $\begin{array}{l}\text { Compromiso para promover el aprendizaje de todos } \\
\text { los estudiantes }\end{array}$ & & \\
\hline $\begin{array}{l}\text { Disposición para promover actitudes y prácticas } \\
\text { democráticas de los estudiantes como ciudadanos } \\
\text { europeos }\end{array}$ & & \\
\hline Actitudes críticas hacia la propia enseñanza & & \\
\hline $\begin{array}{l}\text { Disposición para trabajar en equipo, colaboración y } \\
\text { creación de contactos }\end{array}$ & & \\
\hline
\end{tabular}

Fuente: elaboración propia. 


\section{La propuesta ELITe de áreas de formación del profesorado STEM}

Con la finalidad de atender las necesidades de mejora de competencias detectadas en la tabla 1, se propone una serie de áreas para la formación del profesorado STEM. Estas áreas responden al análisis previo y se implementan en la plataforma digital abierta DojoIBL. En este modelo, se busca establecer mecanismos de interacción con el docente formador y entre el profesorado participante, centrados en la metodología IBL como eje fundamental del modelo de formación. Este modelo se adapta a los contextos educativos actuales, que ubican a los estudiantes en el centro del proceso de aprendizaje y promueven la integración de los distintos estilos de aprendizaje y el desarrollo de competencias. Los módulos propuestos son los siguientes:

- Diseño de buenas actividades IBL basadas en DojoIBL (en detalle en el anexo 1).

- Estrategias de enseñanza utilizando el aprendizaje basado en proyectos.

- Manejo de la diversidad en la educación: diferencias de género, estilos de aprendizaje y personalización.

- Estrategias para introducir problemas socio-científicos en el aula: dilemas, controversias y conversaciones (en detalle en el anexo 1).

- Cómo enfrentar los desafíos y dificultades clave para implementar las actividades de STEM en el aula, utilizando las metodologías de IBL.

- Tecnologías digitales emergentes en la educación STEM: pensamiento computacional, robótica y aprendizaje basado en juegos. 
- Cómo apoyar enfoques STEM neutrales de género en el contexto familiar.

- Cómo superar las experiencias personales de las familias para el éxito en STEM del alumnado.

Estos ocho módulos de formación recogen el modelo ELITe sobre las áreas a desarrollar presentado en la sección anterior y adaptado al contexto español. En dichas áreas se recogen las principales necesidades de formación según el modelo ELITe. Por ejemplo, la importancia del diseño de actividades STEM mediante la plataforma digital Dojorbl indica la importancia que se da, en este modelo, a que el profesor construya sus propios escenarios de aprendizaje basados en IBL. O el uso más intensivo de tecnologías digitales en la educación STEM, que fue identificado como un área a reforzar en la formación del profesorado.

De igual manera, la importancia de introducir temas socio-científicos que promuevan la discusión y la toma de decisiones conjuntas como una manera de ver la ciencia en su contexto y como influencia en nuestra vida cotidiana.

\section{Conclusiones}

El trabajo que se presenta en este capítulo se basa en una premisa fundamental: el desarrollo de las competencias de los docentes es crucial para la reforma educativa y la mejora de los resultados de aprendizaje y los logros de los estudiantes. Esta propuesta ha sido reconocida en el discurso educativo actual en Europa y en otros lugares del mundo (Blömeke, 2017) y está respaldada por los resultados de investigación que apuntan a correlaciones significativas y positivas entre la calidad docente y el rendimiento estudiantil (Caena, 2011).

El desarrollo de la competencia de los docentes STEM, en particular, ha sido un área predominante en el discurso político de la última década y continuará siéndolo en el futuro, en vista de los desafíos a 
los que se enfrentan los docentes de estas áreas para equipar adecuadamente a los futuros ciudadanos e investigadores para comprender las temáticas y controversias científicas y tecnológicas a las que se enfrentará la sociedad, aplicando el conocimiento y las habilidades científicas para formar opiniones basadas en evidencias. El alcance de la exploración de los contextos nacionales es clave para identificar el espacio de intervención de la formación y mejora de las competencias de los docentes STEM.

Por consiguiente, el objetivo principal de este capítulo ha sido desarrollar un marco de competencias de los docentes STEM mediante la metodología IBL, que esté basado en evidencias y que informe a los diseñadores curriculares y responsables de la formación inicial y continua del profesorado sobre cómo actualizar y complementar los actuales programas de formación y actualización docente. Los módulos de formación son además una guía para la actualización de programas de formación, donde la relación entre los nuevos contenidos sociocientíficos con la metodología IBL puede ser un modelo para la mejora de las competencias del profesorado STEM. El hecho de que los módulos se hayan implementado en la plataforma abierta online DojoibL, con una interface para la creación de escenarios por parte del profesor y según la metodología IBL, amplía el impacto en la formación inicial y permanente del profesorado STEM a nivel local e internacional.

\section{Referencias}

Barajas, M., Trifonova, A., Gimenez, J. (2011). The pathway to inquiry-based science education in Europe and beyond: Challenges and solutions for IBSE adoption. En S. Sotiriou y A. Szücs (eds.), Never waste a crisis! Inclusive excellence, innovative technologies and transformed schools as autonomous learning organizations (pp. 1-6). Atenas: European Distance and E-Learning Network. 
Bell, T., Urhahne, D., Schanze, S., y Ploetzner, R. (2010). Collaborative inquiry learning: Models, tools, and challenges. International Journal of Science Education, 32(3), 349-377. doi: 10.1080/09500690802582241 Blömeke, S. (2017), "Modelling teachers" professional competence as a multi-dimensional construct. In Guerriero, S. (ed.), Pedagogical knowledge and the changing nature of the teaching profession. Paris: OECD. Recuperado de https://doi.org/10.1787/9789264270695-7-en

воE. (2013). Ley orgánica 8/2013, de 9 de diciembre, para la mejora de la calidad educativa. BOE-A-2013-12886. Recuperado de https://www.boe. es/eli/es/lo/2013/12/09/8/con (01.07.2019)

воЕ. (2015). Orden ECD/65/2015, de 21 de enero, por la que se describen las relaciones entre las competencias, los contenidos y los criterios de evaluación de la educación primaria, la educación secundaria obligatoria y el bachillerato. Ministerio de Educación, Cultura y Deporte: BOE-A-2015-738. Recuperado de https://www.boe.es/eli/es/o/2015/01/21/ecd65

Caena, F. (2011). Literature review teachers' core competences: Requirements and development. European Commision. Recuperado de https://ec.europa. eu/assets/eac/education/experts-groups/2011-2013/teacher/teacher-competences_en.pdf (01.07.2019)

Comisión Europea. (2015). La profesión docente en Europa: prácticas, percepciones y políticas. Informe de Eurydice. Luxemburgo: Unión Europea.

Corlu, M. S. (2014). Introducing STEM education: Implications for educating our teachers for the age of innovation. Egitim ve Bilim, 39(171), 74-185.

Crawford, B. (2000). Embracing the essence of inquiry: New roles for science teachers. Journal of Research in Science Teaching, 37(9), 916-937.

Cuevas, P., Lee, O., Hart, J., y Deaktor, R. (2005). Improving science inquiry with elementary students of diverse backgrounds. Journal of Research in Science Teaching, 42(3), 337-357.

Deters, K. (2004). Inquiry in the chemistry classroom. The Science Teacher, 71(10), 42-45.

Dick, T. P. y Rallis, S. F. (1991). Factors and influences on high school students' career choices. Journal for Research in Education, 22(4), 281-292.

European Commission. (2007). Science education now: A renewed pedagogy for the future of Europe. Luxembourg: Office for Official Publications of the European Communities. Recuperado de 
https://ec.europa.eu/research/science-society/document_library/pdf_06/ report-rocard-on-science-education_en.pdf

European Commission. (2013). Supporting teacher competence development for better learning outcomes. Education. European Commission. Recuperado de http://ec.europa.eu/dgs/education_culture/repository/education/ policy/school/doc/teachercomp_en.pdf

European Commission. (2015). Science education for responsible citizenship. Luxembourg: European Union. doi:10.2777/12626

Kapelari S., Bonomi C., Dillon, J., Regan, E., Bromley, G., Vergou, A., y Willison, J. (2012). Train the trainer. London: Inquire. Recuperado de http:// www.inquirebotany.org/uploads/files/TTCManualRevisedPrintVersionFINAL.pdf

Lazonder, A. W. y Harmsen, R. (2016). Meta-analysis of inquiry-based learning effects of guidance. Review of Educational Research, 86(3). doi: $10.3102 / 0034654315627366$

Lee, V. S. (2011). The power of inquiry as a way of learning. Innovative Higher Education, 36(3), 149-160.

OECD. (2013). Teaching and Learning International Survey, talis 2013: Conceptual framework, París: OECD. Recuperado de http://www.oecd. org/education/school/TALIs\%20Conceptual\%20Framework_FINAL.pdf OECD. (2018). Teaching and Learning International Survey talis 2018. París: OECD.

Parlamento Europeo (2006). Recomendaciones del Parlamento Europeo y del Consejo de Estado sobre las competencias clave para el aprendizaje permanente, 18 de diciembre (2006/962/CE). Recuperado de http://infofpe.cea.es/fpe/norm/Rec\%2018_2006.pdf

Rossi, A. y Barajas, M. (2015) Elección de estudios Cтıм y desequilibrios de género. Enseñanza de las Ciencias, (33)3, 59-76. doi: https://doi.org/10.5565/ rev/ensciencias.1481

Van Joolingen, W. R., De Jong, T., Lazonder, A. W., Savelsbergh, E. R., y Manlove, S. (2005). Co-lab: Research and development of an online learning environment for collaborative scientific discovery learning. Computers in Human Behavior, 21(4), 671-688. doi: 10.1016/j.chb.2004.10.039 


\section{Anexo 1}

A continuación se presenta el diseño de dos módulos de formación de profesorado STEM mediante la metodología IBL. Ambos han sido implementados en la plataforma DojoIBL. Se puede acceder a cada uno de los módulos mediante el código correspondiente

\section{Módulo 1 \\ Diseño de buenas actividades de IBL basadas en DojoIBL}

Recuperado de http://dojo-ibl.appspot.com/\#/project/5362121193816064 Código: REUFD

El módulo consiste en una introducción al entorno de aprendizaje DojoIBL que permitirá al profesorado STEM abordar, en esta plataforma, los principios básicos para organizar y gestionar la enseñanza-aprendizaje según la metodología IBL, así como sus conceptos centrales. Los participantes podrán experimentar y trabajar en este entorno de aprendizaje de forma individual o con su grupo de trabajo y conseguir así una comprensión profunda de la manera de utilizar los elementos que ofrece Dojoibl para la enseñanza-aprendizaje basado en indagación.

\section{Objetivos de aprendizaje}

El hecho de desarrollar el módulo en el entorno DojoIBL permite a los educadores participantes (además de comprender o profundizar en la metodología de la enseñanza y aprendizaje basado en indagación) utilizar este recurso para diseñar e implementar sus propios escenarios y explicar los conceptos subyacentes en IBL.

Concretamente, se desarrollarán los siguientes contenidos, competencias y actitudes relacionados con el objetivo general mencionado anteriormente. 


\section{Contenidos}

A1. Conocimientos didácticos, comprensión de la metodología de la enseñanza-aprendizaje basado en indagación (IBL); comprensión de los conceptos concretos relacionados con esta metodología, propios de las diferentes materias del ámbito STEM.

A2. Conocimientos sobre el potencial de las TIC y las TAC para facilitar un aprendizaje más efectivo y aportar una herramienta valiosa de gestión de este aprendizaje.

\section{Competencias a desarrollar}

B1. Habilidades metacognitivas y reflexivas durante el propio aprendizaje.

B2. Capacidad de planificar, gestionar y coordinar la enseñanza (en un entorno de enseñanza y aprendizaje virtual).

B3. Habilidad para utilizar materiales didácticos y recursos TIC y TAC. B4. Habilidad para supervisar, adaptar y progresar hacia los objetivos y procesos de enseñanza y aprendizaje.

B5. Capacidad para recolectar, analizar e interpretar evidencias y datos para poder tomar decisiones profesionales con el objetivo de mejorar la enseñanza-aprendizaje propios y el de los estudiantes.

B6. Habilidades de colaboración y trabajo cooperativo (con otros docentes).

\section{Actitudes}

C1. Habilidades de pensamiento crítico hacia las propias capacidades de enseñanza (analizar, discutir, poner en cuestión las prácticas educativas, etc.).

C2. Competencias transversales:

1. Disposición para el trabajo en equipo, para el trabajo colaborativo y para el trabajo colaborativo en red.

2. Sentido de la autoeficacia. 


\begin{tabular}{|c|c|}
\hline Autor & Mario Barajas Frutos, Ricardo Torreblanca Rojas. \\
\hline País & España \\
\hline Organización & Universidad de Barcelona \\
\hline Grupo al que va dirigido & $\begin{array}{l}\text { El escenario busca integrar la participación de los } \\
\text { maestros de sTEM que diseñan actividades basadas } \\
\text { en la indagación con sus estudiantes. }\end{array}$ \\
\hline Resumen & $\begin{array}{l}\text { Este escenario de IBL "Distancia desde la Tierra a } \\
\text { la Luna" tiene como objetivo que los estudiantes } \\
\text { aprendan a encontrar respuestas a preguntas } \\
\text { sencillas, con datos comunes que deben investigarse } \\
\text { en su entorno inmediato, usando herramientas } \\
\text { simples y un método adecuado para lograr con } \\
\text { mayor precisión la respuesta, a la que se llega por } \\
\text { deducción. }\end{array}$ \\
\hline \multirow[b]{2}{*}{ Metas/objetivos } & $\begin{array}{l}\text { El objetivo principal de este escenario es motivar } \\
\text { a los estudiantes a generar su propio método y } \\
\text { modelo para encontrar una respuesta desconocida. } \\
\text { El trabajo en equipo y la participación de todos los } \\
\text { miembros son esenciales para construir una forma } \\
\text { viable de resolver el problema. }\end{array}$ \\
\hline & $\begin{array}{l}\text { Los estudiantes tienen la oportunidad de compartir } \\
\text { posibles soluciones y proponer diferentes métodos } \\
\text { para desarrollar la autonomía, la exploración y la } \\
\text { imaginación de los participantes. Se espera que los } \\
\text { maestros de STEM diseñen actividades que incluyan } \\
\text { contenidos curriculares, en donde apliquen sus } \\
\text { conocimientos previos. Las observaciones registran } \\
\text { y miden el impacto positivo que la actividad tiene } \\
\text { sobre el aprendizaje. }\end{array}$ \\
\hline \multirow{2}{*}{ Estructura/contenidos } & $\begin{array}{l}\text { Se comienza planteando una pregunta para } \\
\text { responder, por ejemplo: ¿cuántos estudiantes de esta } \\
\text { escuela serían necesarios para cubrir la distancia de } \\
\text { la Tierra a la Luna? Luego, se espera a que el grupo } \\
\text { organice la distribución de tareas a realizar. }\end{array}$ \\
\hline & $\begin{array}{l}\text { Para responder la pregunta, los estudiantes tienen } \\
\text { las siguientes pautas: ¿qué datos tienen? } \\
\text { ¿Qué datos necesitan? ¿Cómo pueden conseguirlos? } \\
\text { ¿Qué cálculos deben hacer? ¿Qué instrumentos } \\
\text { deben utilizar (cinta métrica, etc.)? }\end{array}$ \\
\hline
\end{tabular}




\begin{tabular}{|l|l|}
\hline \multirow{7}{*}{ Métodos } & $\begin{array}{l}\text { La estructura para llevar a cabo el escenario IBL } \\
\text { comienza con la formulación de la pregunta inicial: } \\
\text { ¿cuántos estudiantes de esta escuela se necesitarían } \\
\text { para cubrir la distancia de la Tierra a la Luna? }\end{array}$ \\
\hline $\begin{array}{l}\text { A continuación, se espera que los estudiantes } \\
\text { propongan las preguntas pertinentes para resolver } \\
\text { el problema y que mejoren el método seleccionado } \\
\text { para encontrar la respuesta. Durante este proceso, se } \\
\text { establecen los roles de los participantes y los pasos } \\
\text { para resolver la tarea inicial. }\end{array}$ \\
\hline $\begin{array}{l}\text { Finalmente, es importante revisar los datos } \\
\text { recopilados y las herramientas utilizadas para } \\
\text { evaluar el escenario. }\end{array}$ \\
\hline $\begin{array}{l}\text { Después de realizar la implementación, es relevante } \\
\text { la proactividad que los estudiantes adopten ante el } \\
\text { desafío de imaginar, descubrir, analizar y construir } \\
\text { un método adecuado para alcanzar la respuesta } \\
\text { esperada. }\end{array}$ \\
\hline $\begin{array}{l}\text { El desempeño del maestro será una guía que } \\
\text { dé confianza a los participantes y los motive a } \\
\text { profundizar en las diferentes posibilidades para } \\
\text { alcanzar el resultado esperado. }\end{array}$ \\
\hline $\begin{array}{l}\text { Los profesores encuentran en la metodología IBL } \\
\text { estrategias para lograr una participación activa de } \\
\text { los estudiantes. Para esto es importante estimular su } \\
\text { interés y mantener un ambiente de motivación. }\end{array}$ \\
\hline $\begin{array}{l}\text { Por otro lado, la construcción de actividades } \\
\text { basadas en IBL aumenta la propensión a desarrollar } \\
\text { habilidades comunicativas, organizativas, de } \\
\text { investigación y de autoaprendizaje. }\end{array}$ \\
\hline
\end{tabular}




\section{Módulo 2 \\ Estrategias para introducir problemas socio-científicos en el aula: dilemas, controversias y conversaciones}

Recuperado de http://dojo-ibl.appspot.com/\#/inquiry/5723192383504384 Código: EH99J

Este módulo es útil para los profesores de STEM que tienen experiencia utilizando contenidos socio-científicos en el currículo, y quieren explorar cómo aplicarlos en el aula. En este módulo, los profesores mejorarán su conocimiento de estrategias pedagógicas como los dilemas y los grupos de debate; asimismo, desarrollarán sus habilidades para planificar, implementar y reflexionar sobre las actividades del aula en la práctica; y desarrollarán actitudes sobre su función como docentes y la de los estudiantes.

\section{Objetivos generales del módulo}

Se espera que los profesores de STEM desarrollen conocimientos, habilidades y actitudes sobre el uso de las controversias socio-científicas o dilemas en el aula.

\section{Conocimientos}

A1. Desarrollar la comprensión sobre la enseñanza mediante dilemas socio-científicos.

A2. Mejorar la comprensión sobre la propia práctica docente.

\section{Habilidades}

B1. Seleccionar una cuestión socio-científica y planificar un debate para implementar.

B2. Implementar una actividad basada en controversias socio-científicas y debate científico en el aula.

B3. Desarrollar habilidades de indagación sobre la práctica. 


\section{Actitudes}

C1. Percibir la ciencia como una actividad que se realiza a través de la interacción de muchos agentes sociales, no solo los científicos.

C2. Estar cómodo con la idea del profesor como facilitador del conocimiento.

C3. Entender que el alumnado es el responsable de su aprendizaje.

\section{Objetivos específicos}

\section{Contenidos:}

A1a. Definición y objetivos de las cuestiones socio-científicas. A1b. Componentes y ejemplos de cuestiones socio-científicas. A2a. Conocimientos respecto al currículo, las metodologías, los alumnos y la evaluación.

\section{Habilidades:}

B1a. Desarrollar la habilidad de seleccionar/crear una secuencia didáctica basada en una controversia socio-científica y que incluya un debate. B1b. Desarrollar la habilidad de seleccionar recursos para guiar al alumnado hacia los objetivos de aprendizaje.

B2a. Desarrollar habilidades para guiar al alumnado en la resolución de la controversia.

B2b. Desarrollar habilidades para guiar al alumnado en la ejecución del debate.

B3a. Diseñar una estrategia de recogida de datos tanto del alumnado como de mí mismo para recoger datos para mejorar mi práctica. B3b. Desarrollar habilidades de observación para la recogida y el análisis de datos.

B3c. Utilizar los datos recogidos para reflexionar y mejorar la práctica.

\section{Actitudes:}

C1a. Desarrollar una actitud participativa hacia la ciencia.

C1b. Considerar el papel de la ética y los valores en la construcción de conocimiento científico. 
C2a. Entender al profesor como facilitador de los aprendizajes. C2b. Aceptar la incertidumbre de los resultados de la secuencia didáctica.

C3a. Entender el protagonismo del alumnado en el proceso de enseñanza/aprendizaje.

C3b. Desarrollar una actitud positiva hacia el hecho de que los alumnos hablen en el aula.

\begin{tabular}{|l|l|}
\hline Autor & Silvia Alcaraz-Domínguez, Alba Masagué. \\
\hline País & España \\
\hline $\begin{array}{l}\text { Periodo de } \\
\text { implementación }\end{array}$ & Dos semanas \\
\hline Organización & Universidad de Barcelona \\
\hline \multirow{5}{*}{ Rrupo al que va dirigido } & $\begin{array}{l}\text { El escenario está orientado a desarrollar actividades } \\
\text { de aprendizaje basadas en IBL para aspirantes a } \\
\text { profesores de biología. }\end{array}$ \\
\hline \multirow{7}{*}{ Metas/objetivos } & $\begin{array}{l}\text { Este escenario forma parte de las actividades del } \\
\text { Módulo 4: Estrategias para introducir problemas } \\
\text { socio-científicos en el aula: dilemas, controversias y } \\
\text { conversaciones. }\end{array}$ \\
\cline { 2 - 3 } & $\begin{array}{l}\text { El objetivo general es apuntar a los profesores de } \\
\text { biología antes del servicio con el propósito de crear } \\
\text { actividades para que los estudiantes definan una } \\
\text { posición sobre el uso de alimentos transgénicos en el } \\
\text { marco del tema "Herencia genética". }\end{array}$ \\
\hline $\begin{array}{l}\text { La experiencia conecta el módulo didáctico actual, } \\
\text { propuesto en el plan de estudio, con la metodología } \\
\text { IBL. Al mismo tiempo, proporciona una forma } \\
\text { interesante de trabajar en función de la autonomía } \\
\text { de los alumnos y les permite ser críticos y romper } \\
\text { con el esquema habitual de una clase en la que el } \\
\text { profesor expone mientras el alumno escucha. }\end{array}$ \\
\hline
\end{tabular}




\begin{tabular}{|c|c|}
\hline \multirow{3}{*}{ Estructura/contenidos } & $\begin{array}{l}\text { En primer lugar, se presentan brevemente los } \\
\text { contenidos correspondientes al módulo "Herencia } \\
\text { biológica", en el que ya se estaba trabajando. Luego } \\
\text { se hace un acercamiento al problema para que los } \\
\text { estudiantes definan si están de acuerdo o no sobre el } \\
\text { consumo de alimentos transgénicos. }\end{array}$ \\
\hline & $\begin{array}{l}\text { En parejas, los participantes deben establecer } \\
\text { una posición y luego discutirla con otros grupos. } \\
\text { Mientras tanto, la función del maestro es } \\
\text { secundaria, a manera de guía para el proceso de } \\
\text { debate. }\end{array}$ \\
\hline & $\begin{array}{l}\text { La información proporcionada está dirigida a } \\
\text { tomar una decisión clara y objetiva para que los } \\
\text { estudiantes confronten sus propias ideas y dilemas } \\
\text { morales. }\end{array}$ \\
\hline \multirow{3}{*}{ Métodos } & $\begin{array}{l}\text { La primera actividad es la presentación de } \\
\text { información general y útil para que los estudiantes } \\
\text { puedan construir una perspectiva objetiva del uso } \\
\text { de alimentos transgénicos. Luego el grupo se divide } \\
\text { en parejas que definirán su posición y la defenderán } \\
\text { frente al resto del grupo. }\end{array}$ \\
\hline & $\begin{array}{l}\text { Esta actividad tiene la finalidad de generar } \\
\text { controversia y promover un debate posterior en } \\
\text { el que cada participante expondrá argumentos } \\
\text { validados y respaldados. }\end{array}$ \\
\hline & $\begin{array}{l}\text { Por último, se contrastan las ideas y se llega a } \\
\text { conclusiones generales. Además de defender su } \\
\text { posición, las ideas de los otros grupos podrían } \\
\text { integrarse para finalmente proponer alternativas } \\
\text { viables en torno al tema. }\end{array}$ \\
\hline \multirow{3}{*}{ Aprendizajes } & $\begin{array}{l}\text { En este escenario, la participación de los estudiantes } \\
\text { es esencial para alcanzar las metas, mientras que el } \\
\text { maestro solo es una guía durante el proceso. }\end{array}$ \\
\hline & $\begin{array}{l}\text { El pensamiento crítico y la presentación de } \\
\text { argumentos sólidos por parte de los estudiantes es la } \\
\text { base fundamental del trabajo grupal. }\end{array}$ \\
\hline & $\begin{array}{l}\text { El desarrollo de estrategias que cambian el esquema } \\
\text { regular de la clase permite a los estudiantes } \\
\text { involucrarse más en su proceso de aprendizaje y la } \\
\text { experiencia podría ser positiva. }\end{array}$ \\
\hline
\end{tabular}


